\title{
Sulfur Vacancy Clustering and its Impact on Electronic Properties in Pyrite $\mathrm{FeS}_{2}$
}

\author{
Debmalya Ray ${ }^{1}$, Bryan Voigt ${ }^{2}$, Michael Manno ${ }^{2}$, \\ Chris Leighton ${ }^{2}$, Eray S. Aydil ${ }^{2,3}$, Laura Gagliardi ${ }^{*}$ \\ ${ }^{1}$ Department of Chemistry, Minnesota Supercomputing Institute, and Chemical Theory Center, \\ University of Minnesota, 207 Pleasant Street SE, Minneapolis, MN 55455-0431, USA \\ ${ }^{2}$ Department of Chemical Engineering and Materials Science, University of Minnesota, 421 \\ Washington Avenue SE, Minneapolis, MN 55455-0431, USA \\ ${ }^{3}$ Department of Chemical and Biomolecular Engineering, New York University Tandon School \\ of Engineering, 6 Metrotech Center, Brooklyn, NY 11201, USA
}

\section{Table of Contents}

1. Computational Methods 2

2. Figure S1. Comparison of experimental and computed lattice parameter. 3

3. Figure S2. Comparison of experimental and computed Fe-S distance 4

4. Figure S3. Comparison of experimental and computed S-S distance 4

5. Figure S4. Comparison of experimental and computed bandgap 5

6. Finite Temperature and Configurational Entropy Effects on Formation and Binding 5 Energy of Defects

7. Figure S5. Partial charge density of highest occupied crystalline orbital (HOCO) of 9 pyrite supercell containing mono S-vacancy using $\mathrm{PBE}+U(=1.8 \mathrm{eV})$ level of theory

8. Figure S6. Schematic representation of movement of electron from the S-vacancy 10 site to the neighboring $\mathrm{S}$ and Fe sites

9. Figure S7. Comparison of DOS of various charge states of mono S-vacancy 11

10. Figure S8. Band structure and DOS of two S mono-vacancies 12

11. Figure S9. Partial charge density of highest occupied crystalline orbital (HOCO) of 13 pyrite supercell containing S-S dimer vacancy using $\mathrm{PBE}+U(=1.8 \mathrm{eV})$ level of theory 
12. Figure S10. Schematic representation of crystal field theory in presence of S-Sdimer vacancy

13. Figure S11. Band structure and DOS of S-S dimer vacancy 15

14. Figure S12. Band structure and DOS of cis-S di-vacancy 15

15. Figure S13. Band structure and DOS of trans-S di-vacancy 16

16. Figure S14. Band structure and DOS of tetra S-vacancy 16

17. Figure S15. Probability of defect complexation of S-S dimer vacancy as a function 17 of temperature

18. Table S1. Convergence of Total Electronic Energy w.r.t. planewave energy cut off 18

19. Table S2. Convergence of activation energy and defect formation energy w.r.t 18 plane-wave energy cutoff

20. Table S3. Comparison of activation energy w.r.t supercell size and functional 18

21. Table S4. Comparison of defect formation energy w.r.t supercell size and functional 19

22. Table S5. Comparison of binding energy w.r.t supercell size and functional 19

23. Table S6: Comparison of activation energy with and without lattice relaxation. 19

24. Table S7: Effect of zero-point energy and vibration entropy on formation energy 20 and binding energy

25. Table S8. Effect of configurational entropy on formation energy 20

26. Table S9. Comparison of defect formation energy (including configurational entropy 20 only) w.r.t supercell size

27. Table S10. Effect of configurational entropy on binding energy 21

28. Table S11. Comparison of binding energy (including configurational entropy only) 21 w.r.t supercell size

29. Table S12: Comparison of effective mass of holes and electrons using $\mathrm{PBE}+\mathrm{U}\left(=\begin{array}{ll}1.8 & 22\end{array}\right.$ $\mathrm{eV})$ and experiment

30. Table S13: Electronic energy of various structures.

\section{Computational Methods}

Spin-polarized density functional theory (DFT) calculations were performed for a unit cell of pyrite using the Vienna ab-initio Simulation Package (VASP) ${ }^{1-4}$ and projected augmented wave (PAW) potentials. ${ }^{5,6}$ A kinetic energy cutoff of $350 \mathrm{eV}$ and $\Gamma$-centered $7 \times 7 \times 7 \mathrm{k}$-point grid was used for structure optimizations and electronic property calculations. Energy convergence criteria of $10^{-5} \mathrm{eV}$ and force convergence criteria of $0.02 \mathrm{eV} / \mathrm{A}$ were used for all calculations. We tested various DFT functionals such as PBE, ${ }^{7,8} \mathrm{PBE}-\mathrm{D} 3,{ }^{9,10} \mathrm{PBEsol},{ }^{11}$ revPBE, ${ }^{12} \mathrm{PW} 91,{ }^{7} \mathrm{AM} 05,{ }^{13-15}$ $\mathrm{GAM},{ }^{16} \mathrm{TPSS},{ }^{17} \mathrm{PBE}+U(U=1.6,1.8$ and $2.0 \mathrm{eV}),{ }^{7,8,18} \mathrm{HSE06}(a=0.05,0.07,0.10,0.15,0.20$, 
0.25 , where $a$ is the Hartree-Fock exchange parameter) ${ }^{19-21}$ and benchmarked their results with respect to experimental lattice parameter, Fe-S bond distance, S-S bond distance, and band gap. We found that PBE $+U$ with a Hubbard $U$ value of $1.8 \mathrm{eV}$, and the HSE06 functional with $a=0.07$, best describe the structural and electronic properties of pyrite.

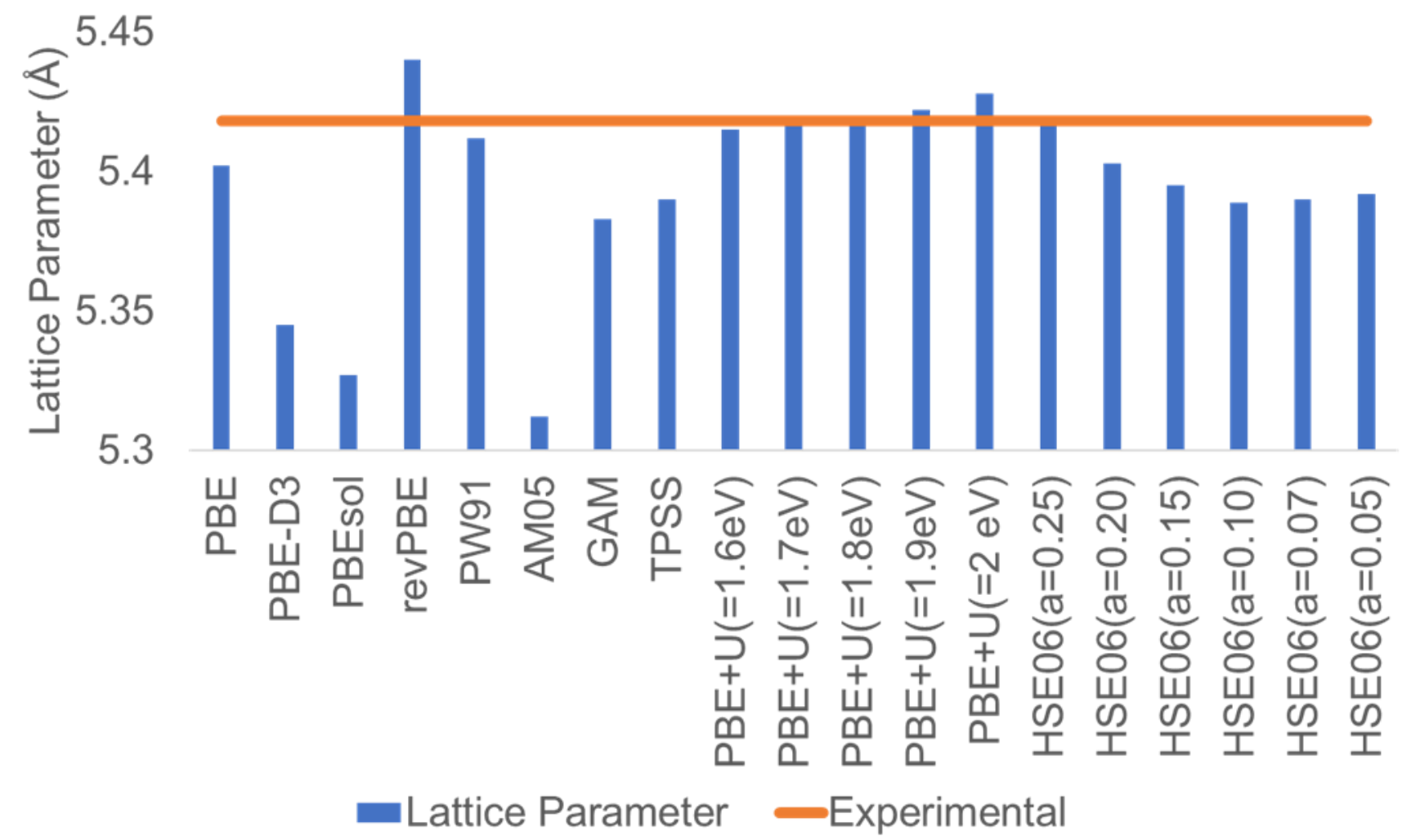

Figure S1. Comparison of experimental and computed pyrite lattice parameter (in $\AA$ ) using various DFT functionals. 


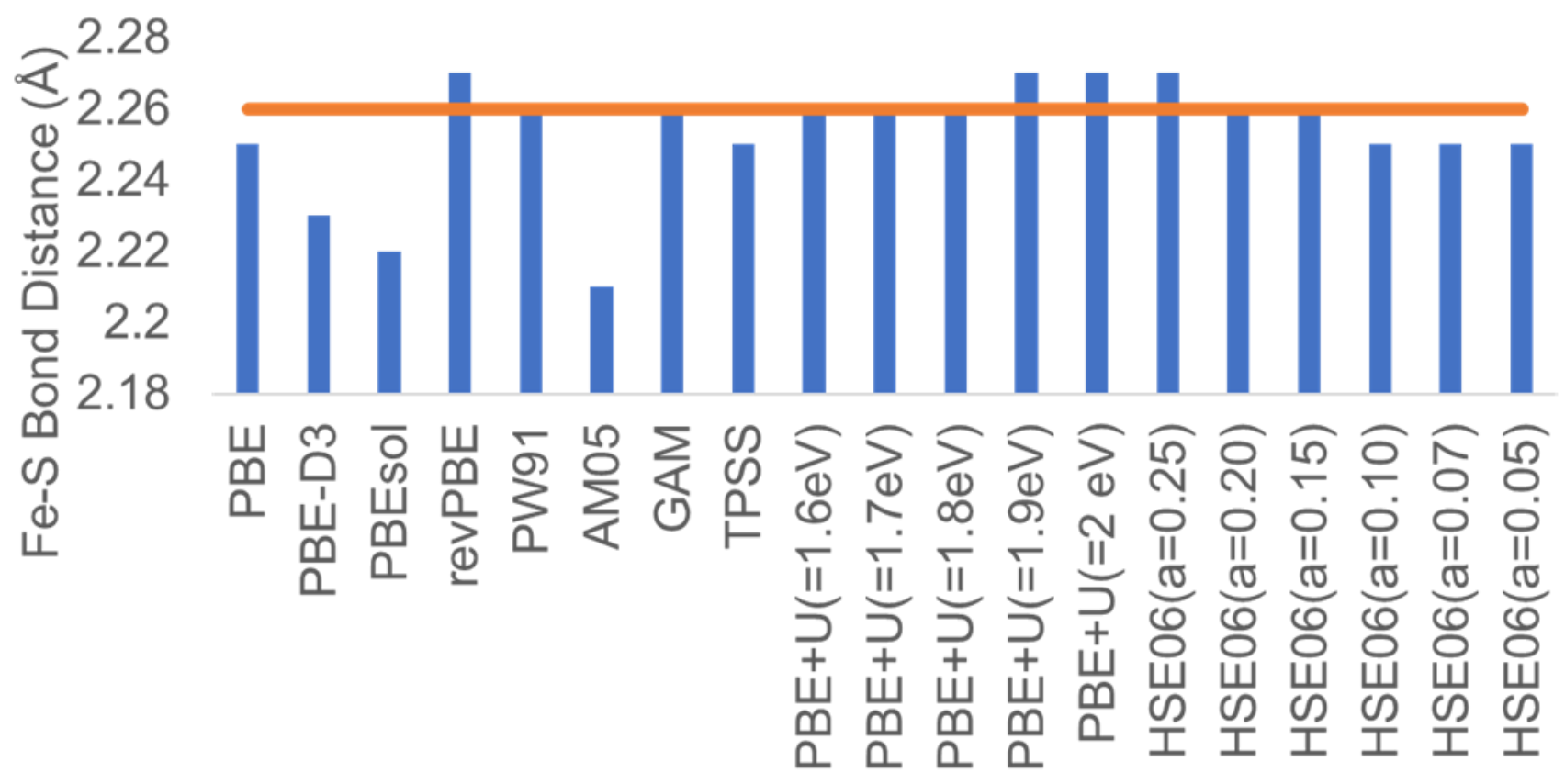

-Fe-S -Experimental

Figure S2. Comparison of experimental and computed pyrite Fe-S bond distance (in $\AA$ ) using various DFT functionals.

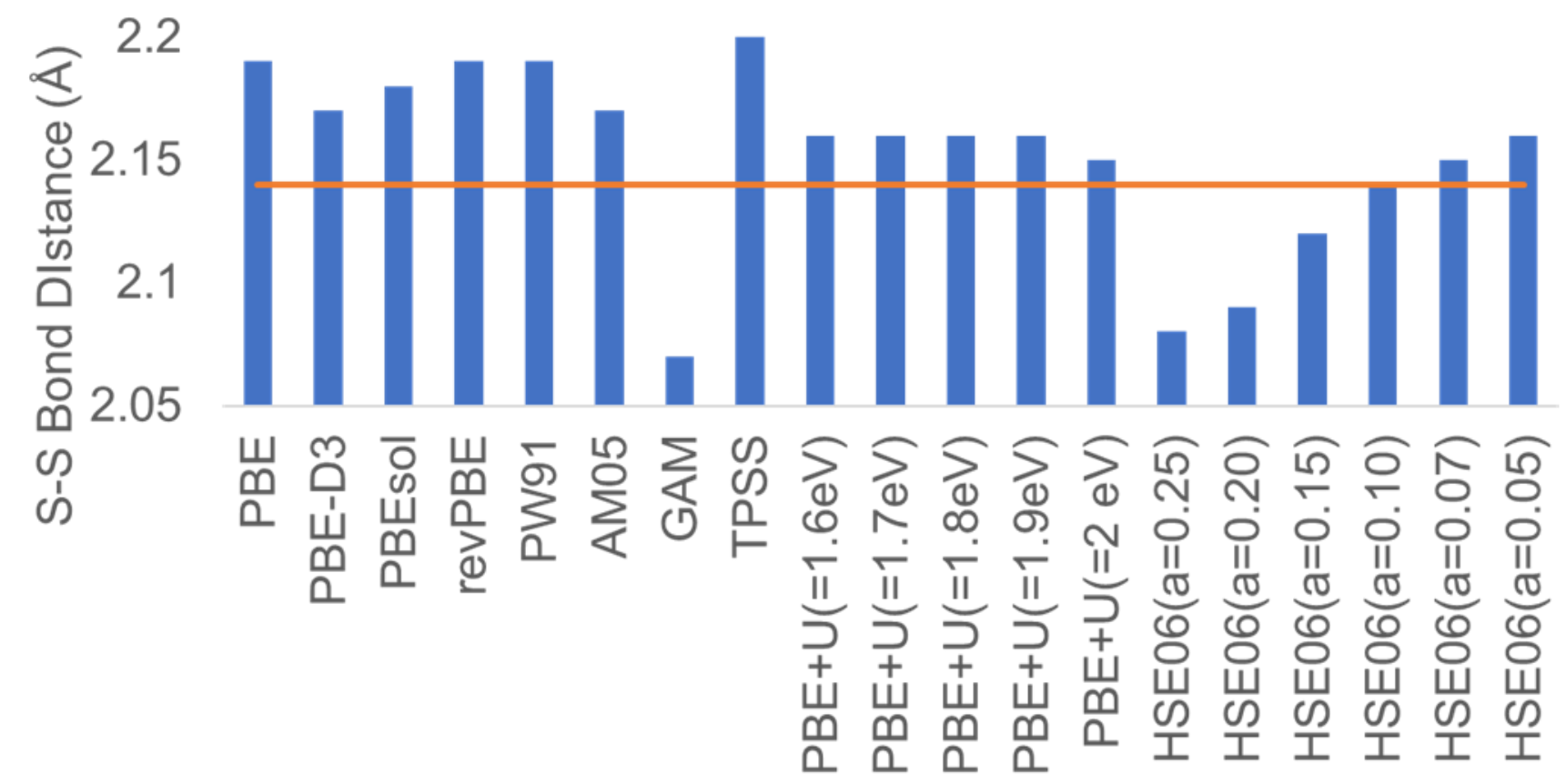

-S-S -Experimental 
Figure S3. Comparison of experimental and computed pyrite S-S bond distance (in $\AA$ ) using various DFT functionals.

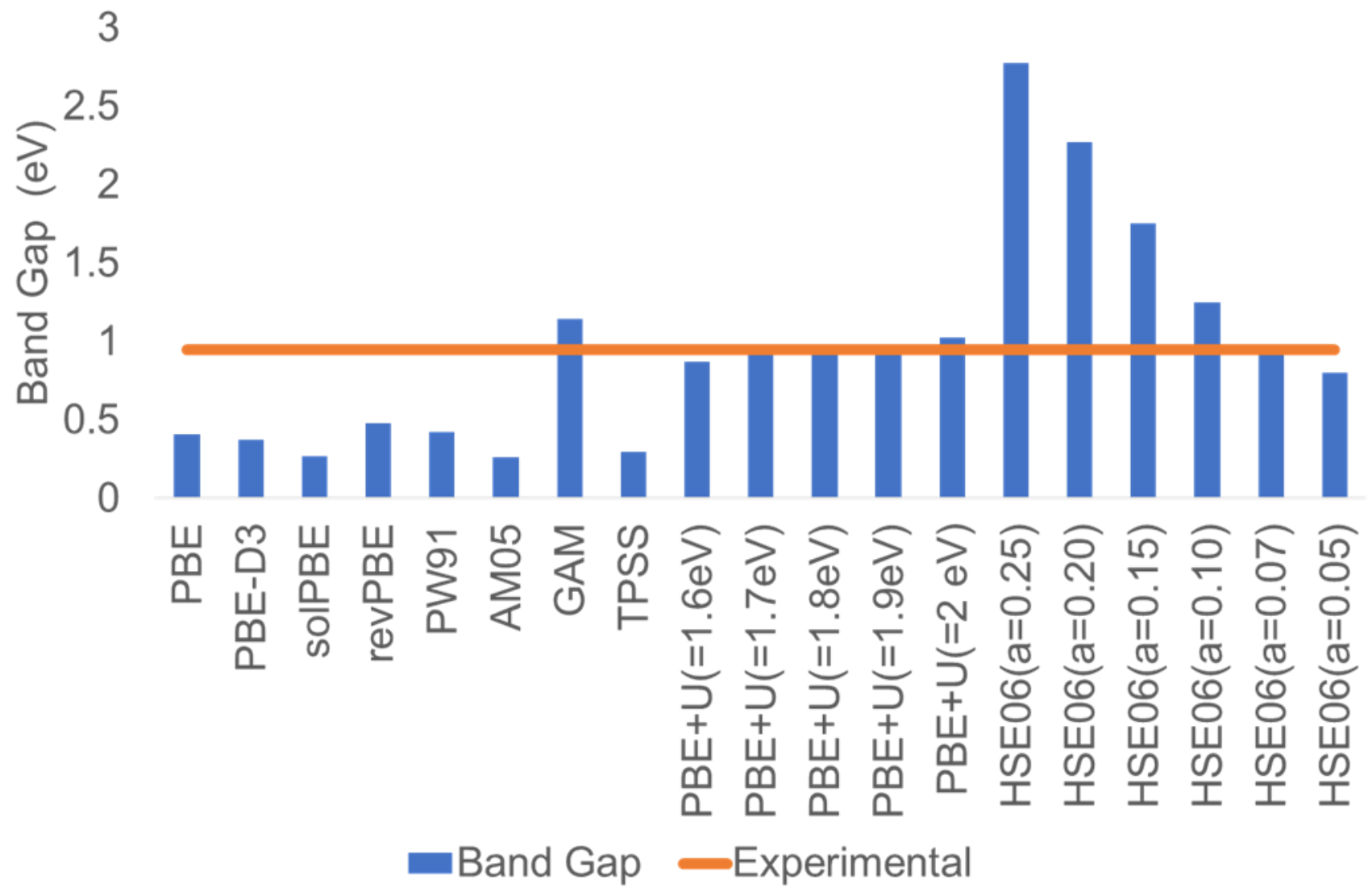

Figure S4. Comparison of experimental and computed band gap (in eV) of pyrite using various DFT functionals.

Effects of Lattice Expansion, Zero-point Energy Corrections, Vibrational Entropy, and Configurational Entropy on Defect Formation and Binding Energies

The total free energy of a system, incorporating effects such as lattice expansion, zero-point energy corrections, vibrational entropy, and configurational entropy, can be written as follows:

$$
G=E+E_{Z P E}-T S_{v i b}-T S_{\text {config }}+\Delta G_{\text {lattice expansion }}(\mathrm{S} 1)
$$


where $G$ is the free energy of the system, $E$ is the electronic energy of the system, $E_{Z P E}$ is the zeropoint energy correction and is defined as $\sum_{i=3 Z} 0.5 \hbar \omega_{i}$ (where $Z$ is the number of atoms in the supercell, $\hbar$ is the reduced Planck constant and $\omega_{i}$ is the vibration frequency), $S_{v i b}$ and $S_{\text {config }}$ are vibrational and configurational entropy, respectively, and $T$ is temperature. $S_{v i b}$ is defined as follows:

$$
S_{v i b}=k_{B} \sum_{i=3 Z-6}\left[\frac{\frac{\hbar \omega_{i}}{k_{B} T}}{\left(\exp ^{\frac{\hbar \omega_{i}}{k_{B} T}}-1\right)}-\ln \left(1-\exp ^{-\frac{\hbar \omega_{i}}{k_{B} T}}\right)\right](\mathrm{S} 2),
$$

and $\Delta G_{\text {lattice expansion }}$ is the total change in vibrational energy due to thermal expansion.

Evaluation of $E_{Z P E}, S_{v i b}$, and $\Delta G_{\text {lattice expansion }}$ all require vibrational frequency calculations. We performed such vibrational frequency calculations using VASP and the density functional perturbation theory (DFPT) ${ }^{22}$ approximation. Due to computational cost, however, we restricted these calculations to $2 \times 2 \times 2$ supercells of defect-free pyrite, the $\mathrm{S}$ mono-vacancy, the S-S dimer and the $\mathrm{S}$ tetra-vacancy.

Incorporating these effects into the defect formation energy (which is calculated from equation 1 of the main text) we can obtain a total free energy for defect formation as

$$
\Delta G_{\text {formation }}=\Delta E_{\text {formation }}+\Delta E_{Z P E}-T \Delta S_{\text {vib }}-T \Delta S_{\text {config }}+\Delta \Delta G_{\text {lattice expansion }}
$$

where $\Delta G_{\text {formation }}$ is the total free energy of defect formation, $\Delta E_{\text {formation }}$ is the defect formation energy without thermal and configurational entropy effects (as calculated by equation 1 in the main text), $\Delta S_{v i b}$ and $\Delta S_{\text {config }}$ are vibrational and configurational entropy corrections, respectively, $T$ is

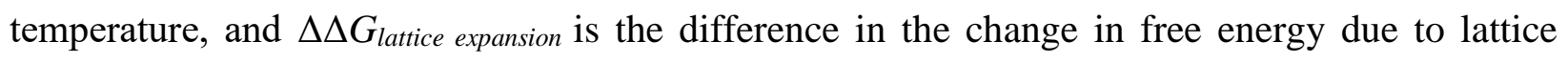
(thermal) expansion for supercells with and without a defect. 
The binding energy (equation 3, main text) will also be affected by incorporating these effects, as

$$
E_{b}=\sum_{i} \Delta G_{\text {formation }}(i)-\Delta G_{\text {formation }}(\text { defect cluster })
$$

where $E_{b}$ is now the total free energy for binding a defect. In the following sections, we will discuss each effect and how it influences $\Delta G_{\text {formation }}$ and $E_{b}$.

\section{(a) Zero-point Energy and Vibrational Entropy}

The free energies of the S mono-vacancy, S-S-dimer vacancy and tetra S-vacancy were calculated at $300 \mathrm{~K}$. Fully relaxed structures (both lattice parameter and atomic positions) were used in the frequency calculations. The effect of zero-point energy and vibrational entropy corrections on the defect formation energy and binding energy of these defects are summarized in Table S6. The zero-point energy and vibrational entropy corrections change the binding energy by less than 0.1 $\mathrm{eV}$. Thus, we ignore these when reporting the binding energy.

\section{(b) Lattice Expansion}

To address this issue, we first started with the DFT-relaxed lattice parameter of supercells containing either the $\mathrm{S}$ mono-vacancy or the S-S dimer vacancy, at $0 \mathrm{~K}$, and used the experimental thermal expansion coefficient ${ }^{23}$ of $4.5 \times 10^{-6} \mathrm{~K}^{-1}$ to get the lattice parameter at $300 \mathrm{~K}$. Vibrational frequencies were calculated using DFPT, and we then took the difference in total vibrational energy between $300 \mathrm{~K}$ and $0 \mathrm{~K}$ to be an energy correction for lattice expansion (i.e., $\Delta G_{\text {lattice }}$ expansion). For both the S mono-vacancy and S-S dimer vacancy, we find $\Delta \Delta G_{\text {lattice expansion }} \approx 2 \mathrm{meV}$, which is significantly lower than the formation and binding energies, and we thus neglect this term when reporting these quantities.

\section{(c) Configurational Entropy}

For a $\mathrm{S}$ mono-vacancy in a given supercell, 


$$
S_{\text {config }}=k_{B} \ln N \quad(\mathrm{~S} 5) \text {, }
$$

where $S_{\text {config }}$ is the configurational entropy and $N$ is the number of equivalent, or possible, defect sites in the pyrite supercell (64 for a $2 \times 2 \times 2$ supercell and 216 for a $3 \times 3 \times 3$ supercell), and $k_{B}$ is Boltzmann constant. Using simple arguments, for two isolated S mono-vacancies we find,

$$
S_{\text {config }}=k_{B} \ln \left(\frac{N(N-1)}{2}\right)(\mathrm{S} 6)
$$

For the cis-S di-vacancy we find

$$
S_{\text {config }}=k_{B} \ln 2 N \quad(\mathrm{~S} 7)
$$

and for the S-S dimer vacancy, trans-S di-vacancy and S tetra-vacancy,

$$
S_{\text {config }}=k_{B} \ln (N / 2) \quad(\mathrm{S} 8)
$$

Thus, at $T=300 \mathrm{~K}$, the change in free energy due to configurational entropy will be $-T S_{\text {config }}$ (see Table S7 for values). The value of the configurational entropy will, of course, depend on the supercell size, as a bigger supercell will have higher configurational freedom for defect formation. Any change in defect formation energy will in turn influence the defect binding energy; these values are reported in Table S8. The overall change in defect formation and binding energies due to configurational entropy effects are reported in Tables $\mathbf{S 9}$ and Table S10, respectively. 


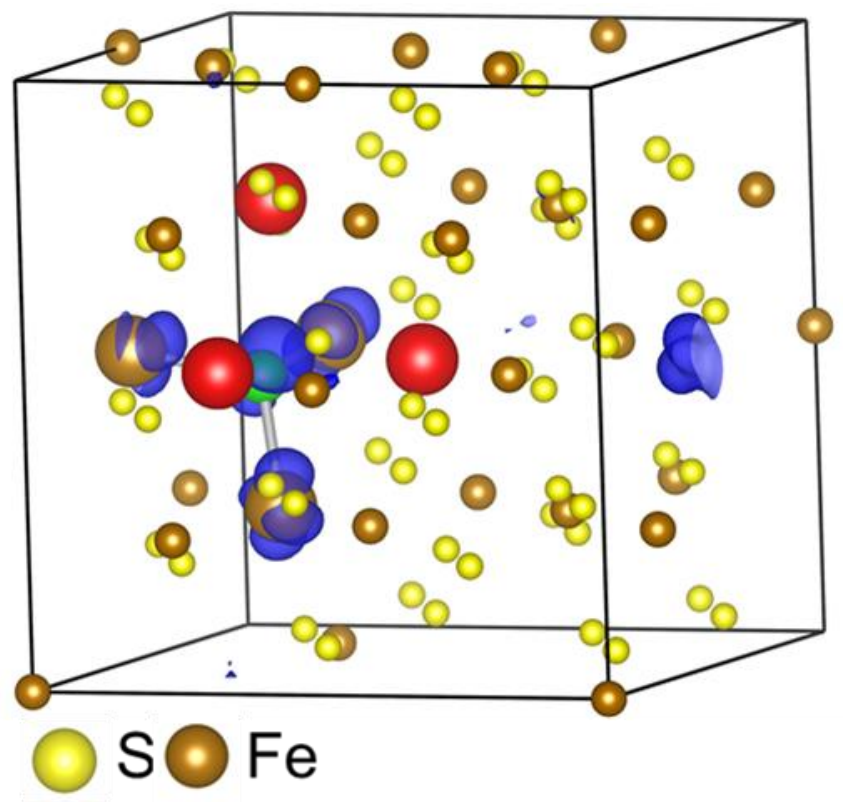

\section{- Fe neighboring the $V_{S}$ \\ $S$ neighboring the $V_{S}$}

Figure S5. Partial charge density (shown in blue) analysis of the highest occupied orbital of a $2 \times 2 \times 2$ pyrite supercell containing one $\mathrm{S}$ mono-vacancy, calculated using the $\mathrm{PBE}+U(U=1.8$ $\mathrm{eV})$ level of theory. 
(a)

$$
s-\longrightarrow v_{s}+e^{+}+1 / 8 s_{8}
$$

(b)
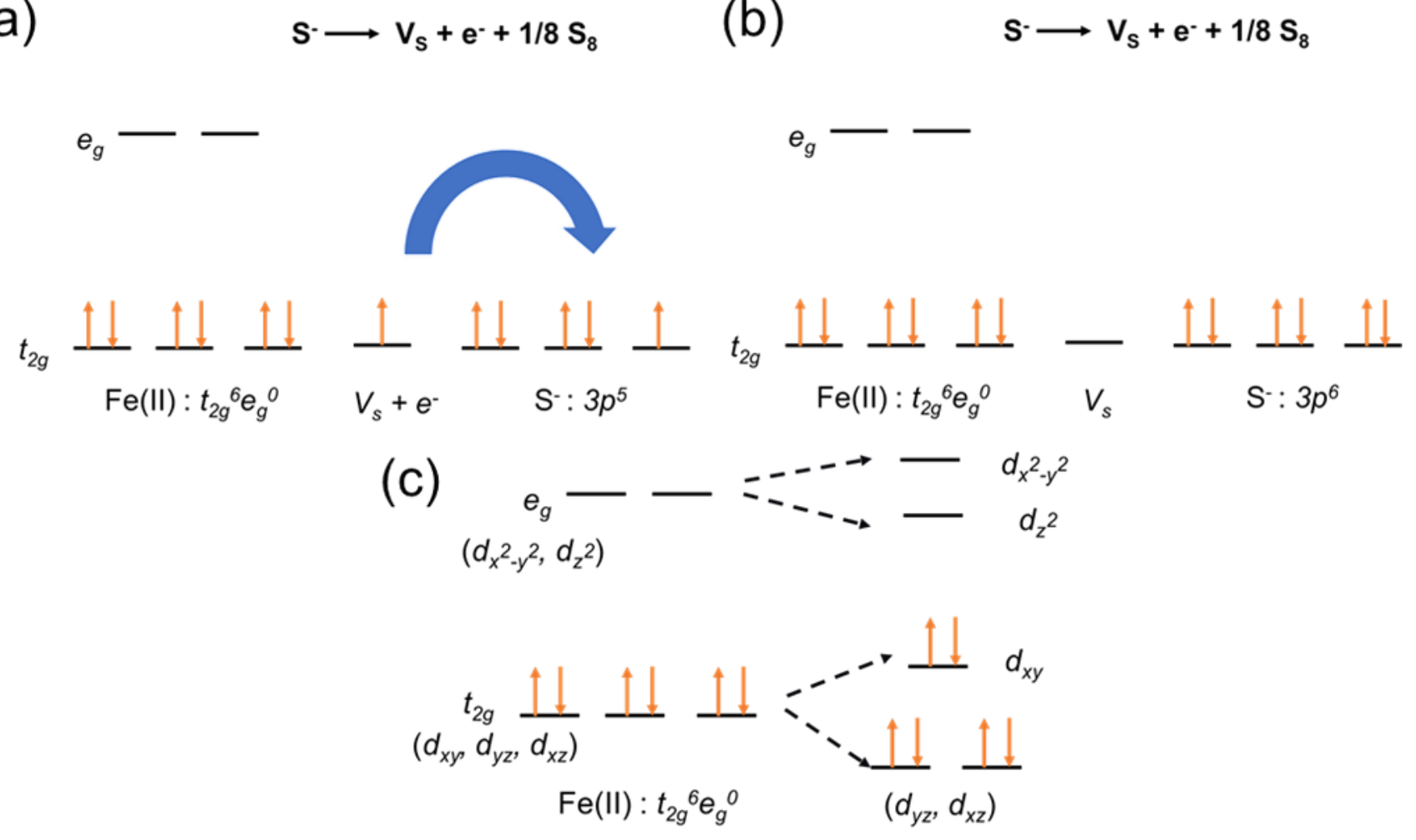

Figure S6. (a-b) Schematic representation of electron transfer from the S mono-vacancy site to the remaining $\mathrm{S}$ in the dimer, effectively doubling its negative charge and, in a simple ionic picture, making it $\mathrm{S}^{2-}$. (c) Schematic representation of how the crystal field splitting of the $3 \mathrm{Fe}$ atoms coordinated to this $\mathrm{S}^{2-}$ atom changes upon introduction of the neighboring $\mathrm{S}$ mono-vacancy. In defect-free pyrite, all Fe atoms are in an octahedral crystal field (left) with the region near the valence band maximum largely derived from Fe $t_{2 g}$ states. Upon introduction of a S mono-vacancy, which induces mild elongation of the $\mathrm{Fe}-\mathrm{S}^{2-}$ bond as the remaining $\mathrm{S}$ moves to occupy the original dimer center-of-mass, these $3 \mathrm{Fe}$ centers are now in a distorted octahedral crystal field (right), creating a $t_{2 g}$-derived donor state close to, but just above, the valence band maximum, thus explaining why the S mono-vacancy produces a very deep donor state in pyrite. 


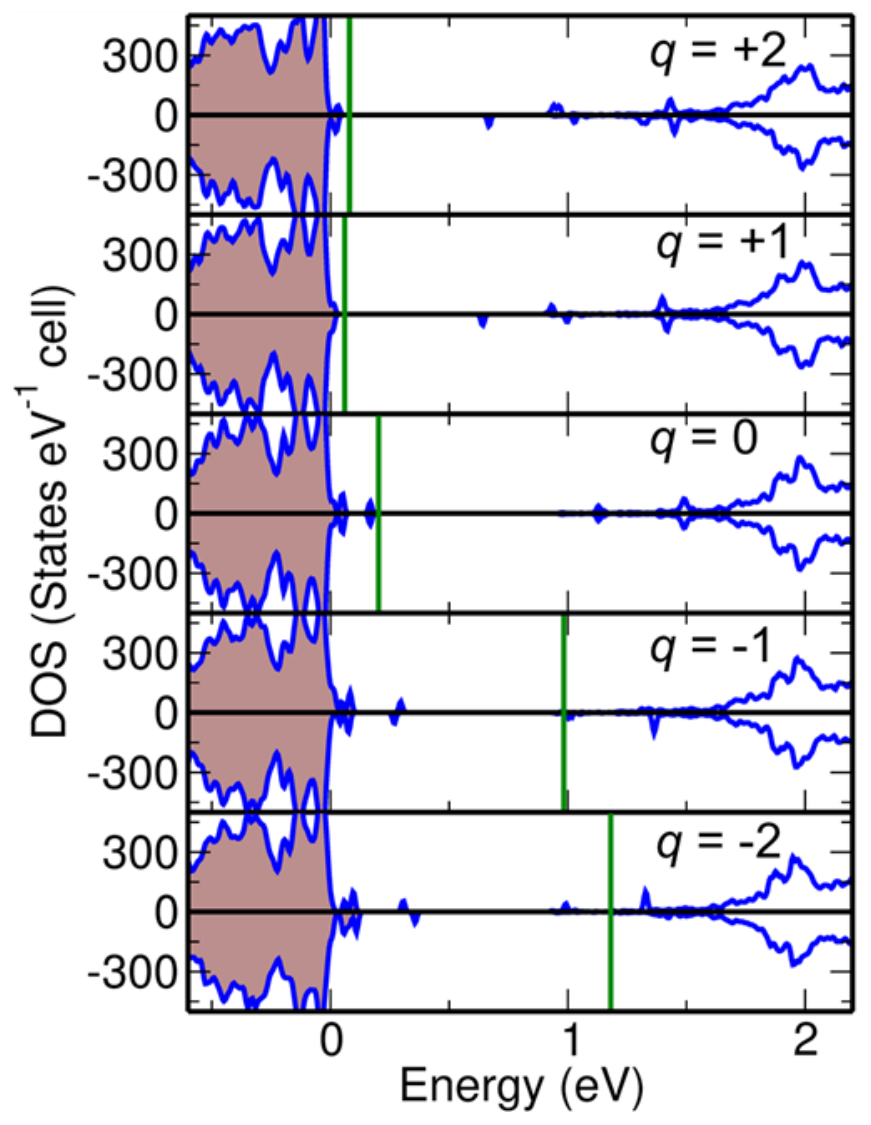

Figure S7. Density-of-states (DOS) comparison of various charge states of the S mono-vacancy in a $3 \times 3 \times 3$ pyrite supercell, calculated using the $\operatorname{PBE}+U(U=1.8 \mathrm{eV})$ level of theory. The vertical green lines represents the Fermi energy. 


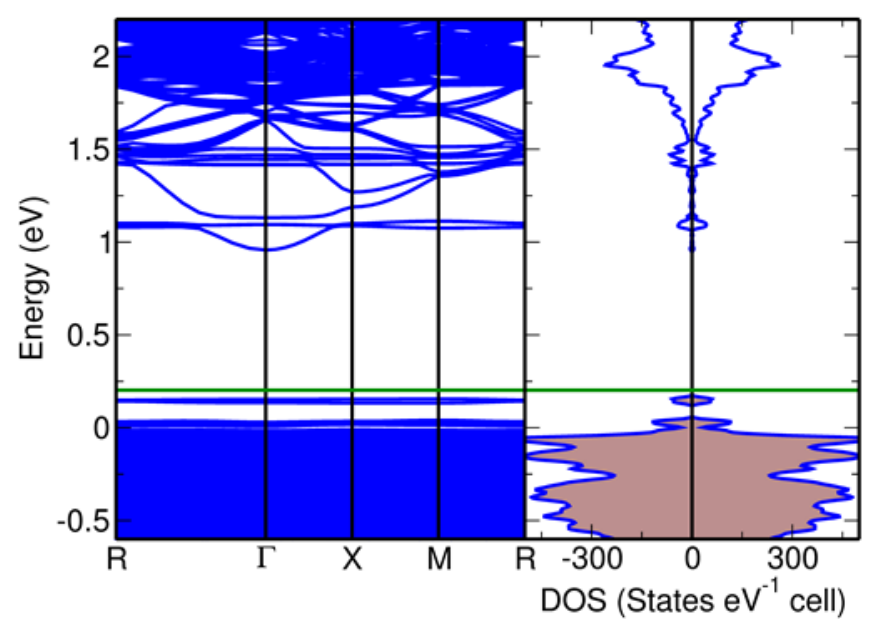

Figure S8. Band structure and DOS of two $\mathrm{S}$ mono-vacancies in a $3 \times 3 \times 3$ pyrite supercell, calculated using $\mathrm{PBE}+U(=1.8 \mathrm{eV})$ level of theory. The horizontal green line represents the Fermi energy. The band structure and DOS are quite similar to that of a single S mono-vacancy, suggesting these two vacancies are essentially non-interacting, and thus this supercell merely contains twice the concentration of defects examined in the case of the single S mono-vacancy. 


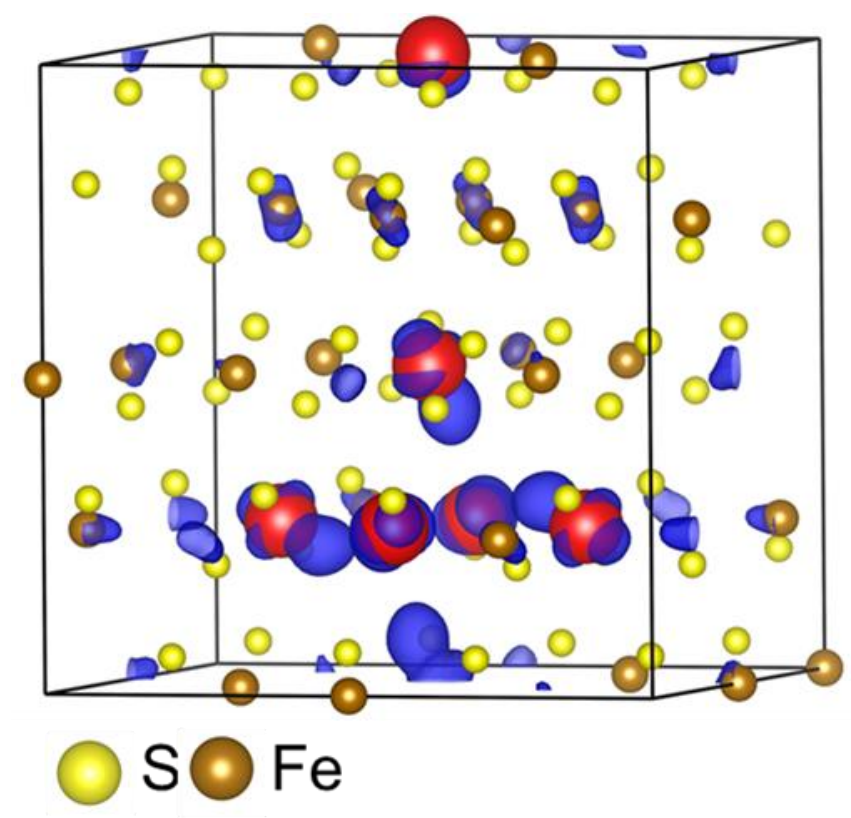

- Fe neighboring the $V_{S}$

Figure S9. Partial charge density (shown in blue) analysis of the highest occupied defect state in a $2 \times 2 \times 2$ pyrite supercell containing a S-S-dimer vacancy, calculated using $\mathrm{PBE}+U(U=1.8 \mathrm{eV})$ level of theory. 
(a) $\quad 2 \mathrm{~S}^{-} \longrightarrow 2 \mathrm{~V}_{\mathrm{S}}+2 \mathrm{e}^{-}+2 / 8 \mathrm{~S}_{8}$

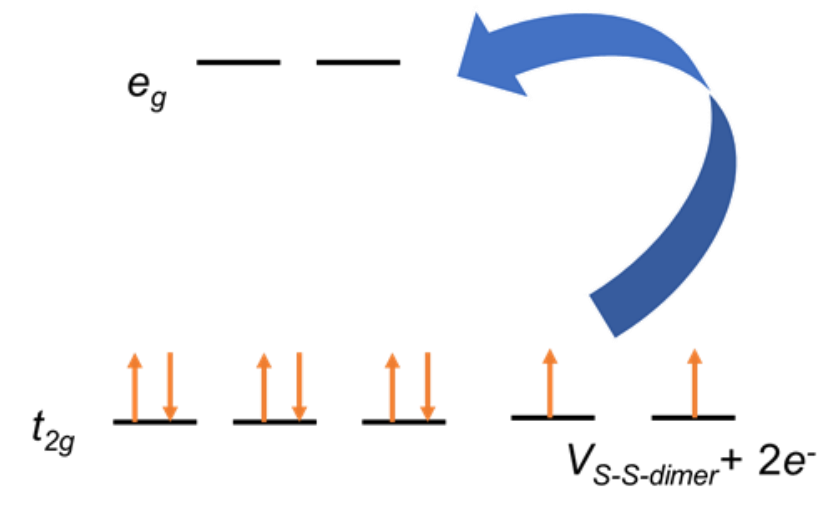

(b) Octahedral Square Pyramidal

Ligand Field Ligand Field
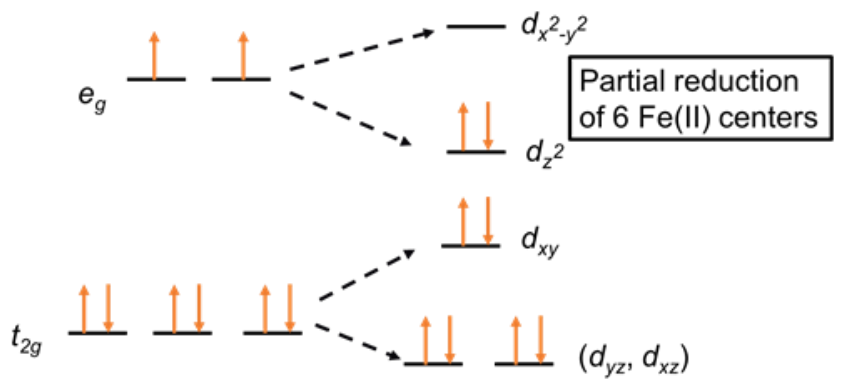

Figure S10. (a) Schematic representing the movement of electrons from the S-S dimer site to the six neighboring Fe centers upon creation of a dimer vacancy. Unlike in the case of the S monovacancy, the S-S-dimer vacancy does not have a nearest-neighbor $\mathrm{S}$ atom where electrons can transfer, thus they move to the six Fe centers coordinated with one of the $\mathrm{S}$ vacancies. (b) Schematic representation of how the crystal field splitting of the six Fe centers reorganizes after a S-S-dimer vacancy is introduced. Due to the creation of the dimer vacancy, these six Fe centers change from octahedral coordination (left) to square planar (right). 


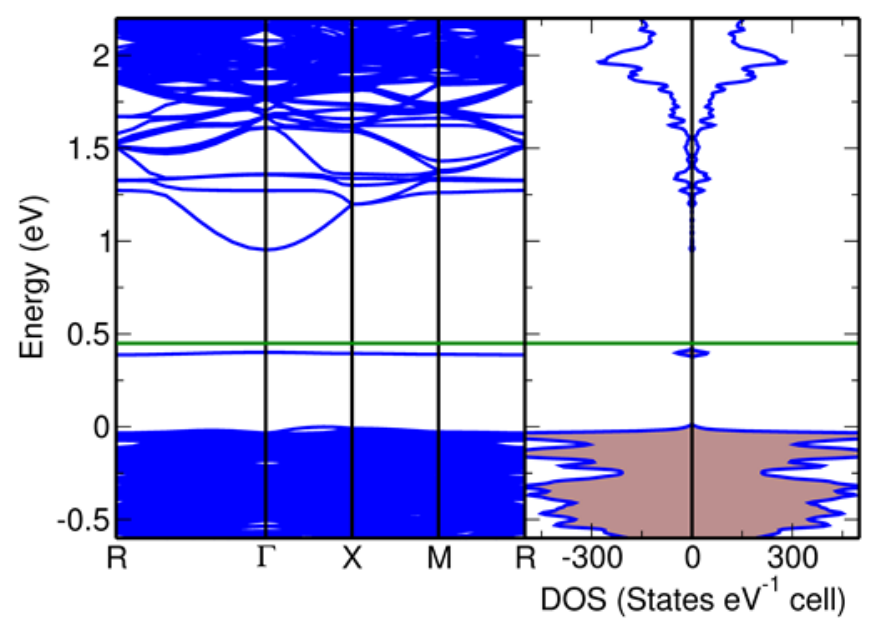

Figure S11. Band structure and DOS of S-S-dimer vacancy in a $3 \times 3 \times 3$ pyrite supercell, calculated using $\mathrm{PBE}+U(U=1.8 \mathrm{eV})$ level of theory. The horizontal green line represents the Fermi energy.

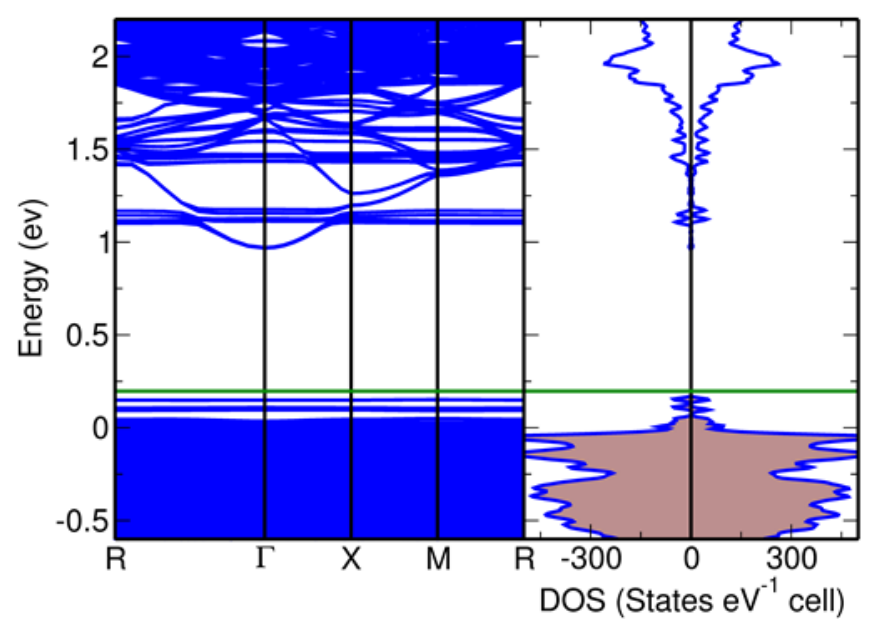

Figure S12. Band structure and DOS of $c i s-\mathrm{S}$ di-vacancy in a $3 \times 3 \times 3$ pyrite supercell calculated, using $\mathrm{PBE}+U(U=1.8 \mathrm{eV})$ level of theory. The horizontal green line represents the Fermi energy. 


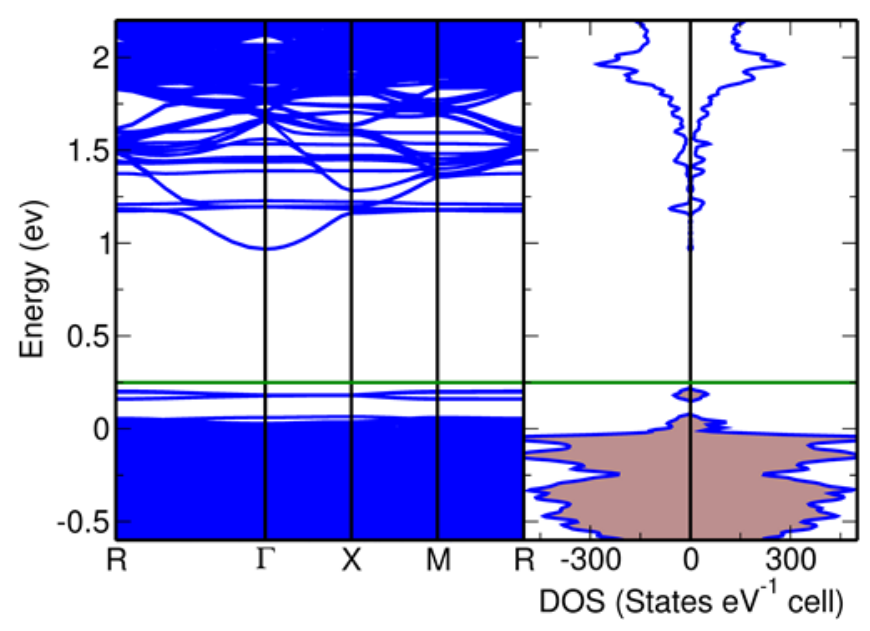

Figure S13. Band structure and DOS of trans-S di-vacancy in a $3 \times 3 \times 3$ pyrite supercell, calculated using $\mathrm{PBE}+U(U=1.8 \mathrm{eV})$ level of theory. The horizontal green line represents the Fermi energy.

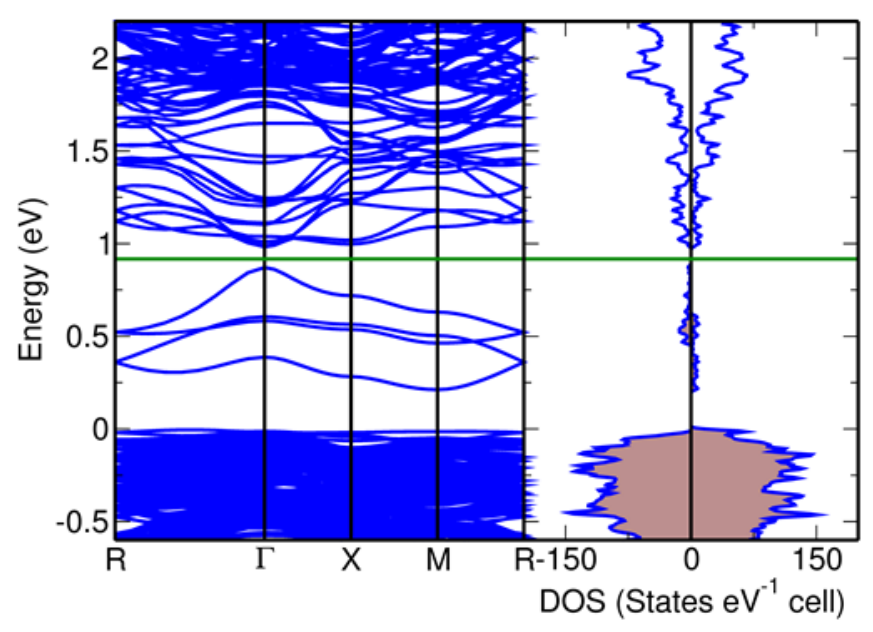

Figure S14. Band structure and DOS of a tetra S-vacancy in a $2 \times 2 \times 2$ pyrite supercell, calculated using $\mathrm{PBE}+U(U=1.8 \mathrm{eV})$ level of theory. The horizontal green line represents the Fermi energy. 


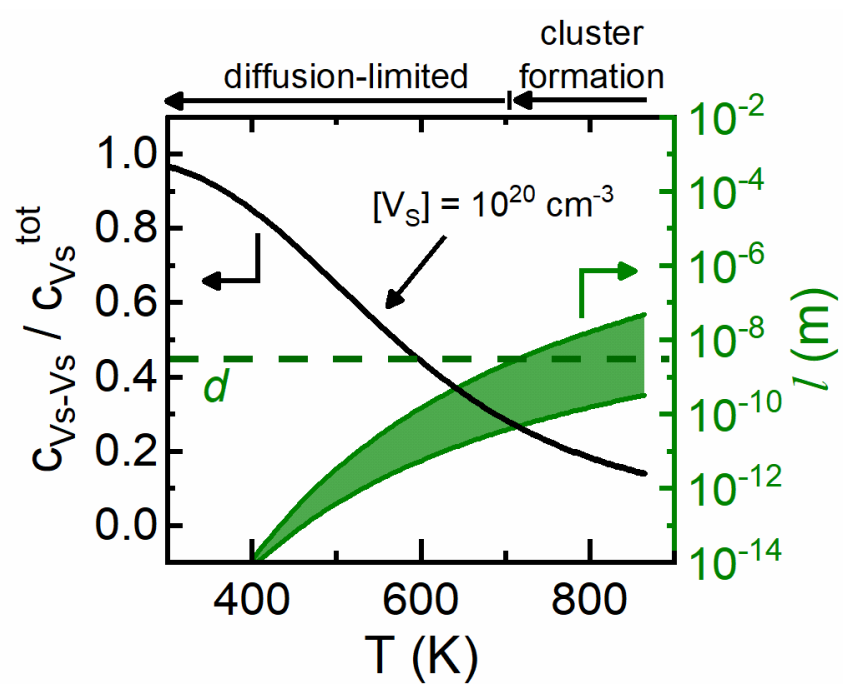

Figure S15. The temperature $(T)$ dependence of (left axis) the fraction of S mono-vacancies $\left(\mathrm{V}_{\mathrm{S}}\right)$ participating in a S-S dimer vacancy for total $\mathrm{S}$ vacancy concentration $10^{20} \mathrm{~cm}^{-3}$, assuming a binding energy of $0.34 \mathrm{eV}$. Also plotted (right axis, green) is the $T$-dependence of a range of $\mathrm{S}$ vacancy diffusion lengths $(l)$ estimated assuming $l=\sqrt{D t}$, where $D$ is the vacancy diffusion coefficient and $t$ is time $(10 \mathrm{~min})$. As upper and lower bounds, DFT-calculated $\mathrm{V}_{\mathrm{S}}$ diffusion ${ }^{24}$ and experimental S self-diffusion coefficients were used, respectively. ${ }^{25}$ The average separation between $\mathrm{V}_{\mathrm{S}}$ (assuming a concentration of $10^{19}-10^{20} \mathrm{~cm}^{-3}$ ) is $2-5 \mathrm{~nm}$, and is marked with a green dashed line. The $T(\sim 710 \mathrm{~K})$ where the range of diffusion lengths falls below this separation distance marks the point where clustering events can no longer occur as the crystal cools, because diffusion is insufficient. 
Table S1: Total electronic energy of the pyrite unit cell with respect to kinetic energy cutoff.

$\begin{array}{ccc}\text { Energy Cutoff } & \text { Total Electronic Energy } & \text { Change in Electronic Energy per } \\ (\mathrm{eV}) & (\mathrm{eV}) & \text { atom }(\mathrm{eV} / \mathrm{atom}) \\ 300 & -64.9324131 & - \\ 325 & -64.9578879 & -0.0021 \\ 350 & -64.9614187 & -0.0003 \\ 375 & -64.9643023 & -0.0002 \\ 400 & -64.9671692 & -0.0002 \\ 425 & -64.9654424 & 0.0001 \\ 450 & -64.9786569 & -0.0011\end{array}$

Table S2: Convergence of activation energy $\left(\Delta E_{\text {activation }}\right)$ and defect formation energy ( $\left.\Delta E_{\text {formation }}\right)$ with respect to kinetic energy cutoff, using a $2 \times 2 \times 2$ supercell and cutoffs of $350 \mathrm{eV}$ and $400 \mathrm{eV}$. The $\Delta E_{\text {formation }}$ reported here does not include contributions from configurational entropy.

$\Delta E_{\text {activation }}$ using $\mathrm{PBE}+U$ and a

Type of Defect

$\begin{array}{ccccc} & 350 \mathrm{eV} & 400 \mathrm{eV} & 350 \mathrm{eV} & 400 \mathrm{eV} \\ \text { S mono-vacancy } & 0.83 & 0.84 & 2.09-2.99 & 2.09-2.99 \\ \text { S-S dimer vacancy } & 0.54 & 0.55 & 3.52-5.52 & 3.50-5.50\end{array}$

Table S3: Comparison of activation energy $\left(\Delta E_{\text {activation }}\right)$ for various defects with respect to supercell size and functional.

$\begin{array}{lccc}\text { Type of Defect } & \Delta E_{\text {activation }} \text { using PBE+U } & \Delta E_{\text {activation }} \text { using PBE+U } & \Delta E_{\text {activation }} \text { using HSE06 } \\ & \text { and a } 3 \times 3 \times 3 \text { supercell } & \text { and a } 2 \times 2 \times 2 \text { supercell } & \text { and a } 2 \times 2 \times 2 \text { supercell } \\ & (\mathrm{eV}) & (\mathrm{eV}) & (\mathrm{eV}) \\ & & & \\ \text { S mono-vacancy } & 0.80 & 0.83 & 0.91 \\ \text { So S mono-vacancies } & 0.80 & 0.78 & 0.86 \\ \text { S-S dimer vacancy } & 0.55 & 0.54 & 0.68 \\ \text { cis-S di-vacancy } & 0.82 & 0.89 & 0.97 \\ \text { trans }- \text { S di-vacancy } & 0.76 & 0.71 & 0.76 \\ \text { S tetra-vacancy } & 0.41 & 0.11 & 0.21\end{array}$


Table S4: Comparison of defect formation energy ( $\left.\Delta E_{\text {formation }}\right)$ for various $S$ vacancies with respect to supercell size and functional. This does not include contributions from configurational entropy.

Type of Defect $\quad \Delta E_{\text {formation }}$ using PBE+U $\quad \Delta E_{\text {formation }}$ using PBE+U $\quad \Delta E_{\text {formation }}$ using HSE06 and a $3 \times 3 \times 3$ supercell and a $2 \times 2 \times 2$ supercell and a $2 \times 2 \times 2$ supercell
$(\mathrm{eV})$
$(\mathrm{eV})$
$(\mathrm{eV})$

\begin{tabular}{|cccc} 
S mono-vacancy & $2.12-3.02$ & $2.09-2.99$ & $2.26-3.16$ \\
Two S mono-vacancies & $4.26-6.06$ & $4.00-5.80$ & $4.26-6.06$ \\
S-S dimer vacancy & $3.74-5.54$ & $3.72-5.52$ & $3.68-5.48$ \\
cis-S di-vacancy & $4.14-5.94$ & $4.10-5.90$ & $4.18-5.98$ \\
trans-S di-vacancy & $3.50-5.30$ & $3.48-5.28$ & $3.84-5.64$ \\
S tetra-vacancy & $6.96-10.56$ & $6.44-10.04$ & $7.20-10.80$
\end{tabular}

Table S5: Comparison of binding energy $\left(E_{b}\right)$ for various defects with respect to supercell size and functional. This does not include contributions from configurational entropy.

Type of Defect $\quad E_{b}$ using $\mathrm{PBE}+\mathrm{U}$ and a $\quad E_{b}$ using $\mathrm{PBE}+\mathrm{U}$ and a $\quad E_{b}$ using HSE06 and a $3 \times 3 \times 3$ supercell $(\mathrm{eV}) \quad 2 \times 2 \times 2$ supercell $(\mathrm{eV}) \quad 2 \times 2 \times 2$ supercell $(\mathrm{eV})$

$\begin{array}{cccc}\text { Two S mono-vacancies } & -0.02 & 0.18 & 0.26 \\ \text { S-S dimer vacancy } & 0.50 & 0.46 & 0.84 \\ \text { cis-S di-vacancy } & 0.10 & 0.08 & 0.34 \\ \text { trans-S di-vacancy } & 0.74 & 0.70 & 0.68 \\ \text { S tetra-vacancy } & 1.52 & 1.92 & 1.84\end{array}$

Table S6: The donor activation energy $\left(\Delta E_{\text {activation }}\right)$ of $\mathrm{S}$ vacancy-related defects, calculated by fixing (at $5.418 \AA$ ) or relaxing the lattice parameter $(a)$. Atomic positions were always relaxed, and the $\mathrm{PBE}+U(U=1.8 \mathrm{eV})$ functional and a $3 \times 3 \times 3$ supercell were used for all calculations.

Defect

$\begin{array}{ccr}\text { Defect } & \Delta E_{\text {activation }} & \Delta E_{\text {activatio }} \\ & \text { when relaxing } a(\mathrm{eV}) & \text { when fixing } \\ \text { S mono-vacancy } & & \\ \text { Two S mono-vacancies } & 0.80 & 0.80 \\ \text { S-S dimer vacancy } & 0.50 & 0.80 \\ \text { cis-S di-vacancy } & 0.82 & 0.55 \\ \text { trans } \text {-S di-vacancy } & 0.76 & 0.82 \\ \text { S tetra-vacancy } & 0.41 & 0.77 \\ \text {. } & & 0.41\end{array}$


Table S7: Effect of zero-point energy and vibrational entropy on the formation energy $\left(\Delta E_{\text {formation }}\right)$ and binding energy $\left(E_{b}\right)$ for various defects using a $2 \times 2 \times 2$ pyrite supercell.

Type of Defect Change in Formation Energy Change in Binding Energy

$(\mathrm{eV})$

$\begin{array}{ccc}\text { S mono-vacancy } & -0.05 & - \\ \text { S-S dimer vacancy } & -0.02 & -0.08 \\ \text { S tetra-vacancy } & -0.16 & -0.04\end{array}$

Table S8: Effect of configurational entropy on the formation energy $\left(\Delta E_{\text {formation }}\right)$ for various defects with respect to supercell size.

$\begin{array}{crc}\text { Type of Defect } & -\mathrm{TS}_{\text {config }}(\mathbf{2} \times \mathbf{2} \times \mathbf{2} \text { supercell })(\mathrm{eV}) & -\mathrm{TS}_{\text {config }}(\mathbf{3} \times \mathbf{3} \times \mathbf{3} \text { supercell })(\mathrm{eV}) \\ \text { S mono-vacancy } & -0.11 & -0.14 \\ \text { Two S mono-vacancies } & -0.20 & -0.25 \\ \text { S-S dimer vacancy } & -0.09 & -0.12 \\ \text { cis-S di-vacancy } & -0.12 & -0.16 \\ \text { trans-S di-vacancy } & -0.09 & -0.12 \\ \text { S tetra-vacancy } & -0.09 & -0.12\end{array}$

Table S9: Comparison of the defect formation energy $\left(\Delta E_{\text {formation }}\right)$ for various defects (incorporating configurational entropy only).

Type of Defect

$$
\begin{gathered}
\Delta G_{\text {formation }} \text { using PBE }+\mathrm{U} \text { and a } \\
3 \times 3 \times 3 \text { supercell }(\mathrm{eV})
\end{gathered}
$$

S mono-vacancy

$1.98-2.88$

4.01-5.81

$3.62-5.42$

$3.98-5.78$

$3.38-5.18$

6.84-10.44
$\Delta G_{\text {formation }}$ using $\mathrm{PBE}+\mathrm{U}$ and a

$2 \times 2 \times 2$ supercell $(\mathrm{eV})$

$1.98-2.88$

$3.80-5.60$

$3.63-5.43$

3.98-5.78

3.39-5.19

$6.35-9.95$ 
Table S10: Effect of configurational entropy on binding energy $\left(E_{b}\right)$ for various defects with respect to supercell size.

$\begin{array}{ccc}\text { Type of Defect } & \text { Decrease in binding energy } & \text { Decrease in binding energy } \\ & (\mathbf{2} \times \mathbf{2} \times \mathbf{2} \text { supercell })(\mathrm{eV}) & (\mathbf{3} \times \mathbf{3} \times \mathbf{3} \text { supercell })(\mathrm{eV}) \\ \text { Two S mono-vacancies } & -0.02 & -0.03 \\ \text { S-S dimer vacancy } & -0.13 & -0.16 \\ \text { cis-S di-vacancy } & -0.10 & -0.12 \\ \text { trans-S di-vacancy } & -0.13 & -0.16 \\ \text { S tetra-vacancy } & -0.35 & -0.44\end{array}$

Table S11: Comparison of binding energy $\left(E_{b}\right)$ for various defects (incorporating configurational entropy only).

\begin{tabular}{ccc} 
Type of Defect & \multicolumn{2}{c}{$E_{b}$ using PBE+U and a } \\
& $3 \times 3 \times 3$ supercell $(\mathrm{eV})$ & $E_{b}$ using $\mathrm{PBE}+\mathrm{U}$ and a \\
Two S mono-vacancies & -0.05 & 0.16 \\
S-S dimer vacancy & 0.34 & 0.33 \\
cis-S di-vacancy & -0.02 & -0.02 \\
trans-S di-vacancy & 0.58 & 0.57 \\
S tetra-vacancy & 1.08 & 1.57
\end{tabular}


Table S12: Comparison of $\mathrm{PBE}+U(U=1.8 \mathrm{eV})$-calculated effective masses with past theory and experiment.

$\begin{array}{cccc}\text { Effective Mass } & \text { This Work } & \text { Previous Theoretical } & \text { Experimental Results } \\ & & \text { Work } & \\ \text { Holes } & \begin{array}{c}1.46(\mathrm{VBM} \rightarrow \Gamma) \\ 1.95(\mathrm{VBM} \rightarrow \mathrm{X})\end{array} & 1.23-1.98^{26} & 2.2 \pm 0.7^{27} \\ \text { Electrons } & 0.56 & 0.49^{26} & 0.45^{28,29}\end{array}$

Table S13: Total electronic energies (eV) of computed structures

$\begin{array}{ccc}\text { Total Electronic Energy } & \text { Total Electronic Energy } & \text { Total Electronic Energy } \\ \text { using PBE+U and a } & \text { using PBE+U and a } & \text { using HSE06 and a } \\ 3 \times 3 \times 3 \text { supercell }(\mathrm{eV}) & 2 \times 2 \times 2 \text { supercell }(\mathrm{eV}) & 2 \times 2 \times 2 \text { supercell }(\mathrm{eV}) \\ & & \\ -1754.047235 & -519.603295 & -618.986047 \\ -1746.927456 & -512.510751 & -611.399734 \\ -1739.793553 & -505.516109 & -604.085282 \\ -1740.311506 & -505.872183 & -604.652882 \\ -1739.900072 & -505.511025 & -604.152399 \\ -1740.537790 & -506.121308 & -604.485547 \\ -1727.092876 & -493.139507 & -590.496369\end{array}$

\section{Supporting Information References}

(1) Kresse, G.; Furthmüller, J. Efficient Iterative Schemes for Ab Initio Total-Energy

Calculations Using a Plane-Wave Basis Set. Phys. Rev. B 1996, 54, 11169.

(2) Kresse, G.; Furthmüller, J. Efficiency of Ab-Initio Total Energy Calculations for Metals and Semiconductors Using a Plane-Wave Basis Set. Comput. Mater. Sci. 1996, 6, 15.

(3) Kresse, G.; Hafner, J. Ab Initio Molecular Dynamics for Liquid Metals. Phys. Rev. B 1993, 47, 558. 
(4) Kresse, G.; Hafner, J. Ab Initio Molecular-Dynamics Simulation of the Liquid-MetalAmorphous-Semiconductor Transition in Germanium. Phys. Rev. B 1994, 49, 14251.

(5) Blöchl, P. E. Projector Augmented-Wave Method. Phys. Rev. B 1994, 50, 17953.

(6) Kresse, G.; Joubert, D. From Ultrasoft Pseudopotentials to the Projector AugmentedWave Method. Phys. Rev. B 1999, 59, 1758.

(7) Perdew, J. P.; Wang, Y. Accurate and Simple Analytic Representation of the Electron-Gas Correlation Energy. Phys. Rev. B 1992, 45, 13244.

(8) Perdew, J. P.; Burke, K.; Ernzerhof, M. Generalized Gradient Approximation Made Simple. Phys. Rev. Lett. 1996, 77, 3865.

(9) Grimme, S.; Antony, J.; Ehrlich, S.; Krieg, H. A Consistent and Accurate Ab Initio Parametrization of Density Functional Dispersion Correction (DFT-D) for the 94 Elements H-Pu. J. Chem. Phys. 2010, 132, 154104.

(10) Becke, A. D.; Johnson, E. R. A Density-Functional Model of the Dispersion Interaction. $J$. Chem. Phys. 2005, 123, 154101.

(11) Perdew, J.; Ruzsinszky, A.; Csonka, G.; Vydrov, O.; Scuseria, G.; Constantin, L.; Zhou, X.; Burke, K. Restoring the Density-Gradient Expansion for Exchange in Solids and Surfaces. Phys. Rev. Lett. 2008, 100, 136.

(12) Zhang, Y.; Yang, W. Comment on "Generalized Gradient Approximation Made Simple. Phys. Rev. Lett. 1998, 80, 890.

(13) Mattsson, A. E.; Armiento, R. Implementing and Testing the AM05 Spin Density Functional. Phys. Rev. B 2009, 79, 1. 
(14) Mattsson, A. E.; Armiento, R.; Paier, J.; Kresse, G.; Wills, J. M.; Mattsson, T. R. The AM05 Density Functional Applied to Solids. J. Chem. Phys. 2008, 128, 084714.

(15) Armiento, R.; Mattsson, A. E. Functional Designed to Include Surface Effects in SelfConsistent Density Functional Theory. Phys. Rev. B 2005, 72, 1.

(16) Yu, H. S.; Zhang, W.; Verma, P.; He, X.; Truhlar, D. G. Nonseparable ExchangeCorrelation Functional for Molecules, Including Homogeneous Catalysis Involving Transition Metals. Phys. Chem. Chem. Phys. 2015, 17, 12146.

(17) Sun, J.; Marsman, M.; Csonka, G. I.; Ruzsinszky, A.; Hao, P.; Kim, Y. S.; Kresse, G.; Perdew, J. P. Self-Consistent Meta-Generalized Gradient Approximation within the Projector-Augmented-Wave Method. Phys. Rev. B 2011, 84, 1.

(18) Rohrbach, A.; Hafner, J.; Kresse, G. Electronic Correlation Effects in Transition-Metal Sulfides. J. Phys.: Condens. Matter 2003, 15, 979.

(19) Heyd, J.; Scuseria, G. E.; Ernzerhof, M. Erratum: Hybrid Functionals Based on a Screened Coulomb Potential (Journal of Chemical Physics (2003) 118 (8207)). J. Chem. Phys. 2006, 124, 219906.

(20) Heyd, J.; Peralta, J. E.; Scuseria, G. E.; Martin, R. L. Energy Band Gaps and Lattice Parameters Evaluated with the Heyd-Scuseria-Ernzerhof Screened Hybrid Functional. $J$. Chem. Phys. 2005, 123, 174101.

(21) Heyd, J.; Scuseria, G. E. Assessment and Validation of a Screened Coulomb Hybrid Density Functional. J. Chem. Phys. 2004, 120, 7274.

(22) Gonze, X.; Lee, C. Dynamical Matrices, Born Effective Charges, Dielectric Permittivity 
Tensors, and Interatomic Force Constants from Density-Functional Perturbation Theory. Phys. Rev. B 1997, 55, 10355.

(23) Willeke, G.; Blenk, O.; Kloc, C.; Bucher, E. Preparation and Electrical Transport Properties of Pyrite $\left(\mathrm{FeS}_{2}\right)$ Single Crystals. J. Alloys Compd. 1992, 178, 181.

(24) Zhang, Y. N.; Law, M.; Wu, R. Q. Atomistic Modeling of Sulfur Vacancy Diffusion Near Iron Pyrite Surfaces. J. Phys. Chem. C 2015, 119, 24859.

(25) Watson, E. B.; Cherniak, D. J.; Frank, E. A. Retention of Biosignatures and MassIndependent Fractionations in Pyrite: Self-Diffusion of Sulfur. Geochim. Cosmochim. Acta 2009, 73, 4792 .

(26) Hu, J.; Zhang, Y.; Law, M.; Wu, R. First-Principles Studies of the Electronic Properties of Native and Substitutional Anionic Defects in Bulk Iron Pyrite. Phys. Rev. B 2012, 85, 085203.

(27) Altermatt, P. P.; Kiesewetter, T.; Ellmer, K.; Tributsch, H. Specifying Targets of Future Research in Photovoltaic Devices Containing Pyrite $\left(\mathrm{FeS}_{2}\right)$ by Numerical Modelling. Sol. Energy Mater. Sol. Cells 2002, 71, 181.

(28) Karguppikar, A. M.; Vedeshwar, A. G. Transport Properties of Thin Iron Pyrite Films. Phys. Status Solidi A 1986, 95, 717.

(29) Tsay, M.-Y.; Huang, Y.-S.; Chen, Y.-F. Photoconduction of Synthetic Pyrite FeS 2 Single Crystals. J. Appl. Phys. 1993, 74, 2786. 